\title{
Cobertura del Dorso de los Dedos de la Mano Con Colgajos Tipo Propeller de Perforantes de Arteria Intermetacarpiana Dorsal
}

\section{Covering Dorsal Defects in the Fingers of the Hand with Intermetacarpal Artery Perforator Flaps in Propeller Style}

\author{
Arturo Vela-Lasagabaster ${ }^{1}$ Ana De Juan-Huelves ${ }^{1}$ Laura Gutiérrez-Roca ${ }^{1}$ \\ ${ }^{1}$ Cirugía Plástica y Reparadora, Hospital Universitario Ramón y Cajal, \\ Madrid, Spain \\ Address for correspondence Arturo Vela Lasagabaster, MD, Cirugía \\ Plástica y Reparadora, Hospital Universitario Ramón y Cajal, Madrid, \\ Spain (e-mail: arturovelasaga@gmail.com).
}

Rev Iberam Cir Mano

\section{Resumen \\ Palabras clave \\ - lesiones de la Mano \\ - colgajos de perforantes \\ - colgajos en isla}

Las lesiones en el dorso los dedos de la mano, debidas a traumatismos, infecciones o quemaduras, son causa frecuente de exposición de estructuras nobles óseas, articulares y/o tendinosas. El dorso de la mano ofrece la posibilidad de cobertura donante con una gran similitud de calidad de tejido. Por ello, una opción sencilla y práctica es la cobertura con un colgajo tipo Propeller de perforantes de la arteria intermetacarpiana dorsal, conocido en la literatura médica anglosajona como "Quaba flap," y sus variantes. Presentamos una serie de 3 pacientes, con afectación y exposición noble del dorso de los dedos, desde F1, PIP Y F2, ocurridos tras infección, quemadura y traumatismo, respectivamente, que se vieron beneficiados de esa técnica, empleando, en cada caso, un colgajo de distinto espacio intermetacarpiano dorsal. Especificamos los detalles de la técnica quirúrgica con referencias anatómicas.

All injuries at the back of the fingers, due to a trauma, infections or burns, are frequently caused by the exposure of noble osseous structures, articular and/or tendons. The back of the hand offers the possibility of donor coverage with a great similarity of tissue quality. Therefore, a simple and practical option is the coverage with a dorsal intermetacarpal artery perforators Propeller flap, known in the English medical literature as "Quaba flap," and its variants. The purpose of this article is to try to show the suitability of the IMCAP perforator flap in the covering of defects on the back of the fingers of the hand. So, we present a series of three patients, with a noble involvement and exposure of the back of the fingers, from F1, PIP and F2, occurring after an infection, a burnt and a trauma, respectively, being benefited from this technique, using, in each case, a flap from a different dorsal intermetacarpal space. We specify the details of this surgical technique with different anatomical references. received

June 6, 2019

accepted

September 24, 2019
DOI https://doi.org/

10.1055/s-0039-3399515. ISSN $1698-8396$.
Copyright $\odot$ Thieme Revinter

Publicações Ltda, Rio de Janeiro, Brazil
License terms

(c) (i) $\ominus$ (\$) 


\section{Introducción}

El área anatómica del dorso de los dedos es una zona que, por sus prominencias articulares, puede afectarse, con más frecuencia, en los traumatismos, infecciones o quemaduras de la mano, dejando expuestas estructuras subyacentes nobles: óseas, articulares $\mathrm{y} / \mathrm{o}$ tendinosas, que precisan de una cobertura preferente y con tejido bien vascularizado. Existen múltiples opciones para ello, colgajos en bandera, colgajo de dedos cruzados heterodigital, colgajos en isla a flujo reverso homodigital, etc. ${ }^{1,2}$ Sin embargo, la cobertura con colgajos del dorso de la mano ofrece una calidad similar al tejido perdido. ${ }^{3}$

Presentamos 3 casos clínicos diferentes en cuanto a etiología y zona de exposición noble, pero similares en su resolución quirúrgica, para cobertura de primera falange (F1), articulación interfalángica proximal (PIP), segunda falange (F2) y articulación intermetacarpiana distal (DIP). Los 3 defectos son cubiertos empleando colgajos tipo Propeller de perforantes comisurales de la arteria metacarpiana dorsal, cada uno de un espacio intermetacarpiano diferente.

\section{Introducción Anatómica}

La arteria radial se introduce en la mano por su cara dorsolateral, por encima y medial a la articulación trapeciometacarpiana, para hacerse volar y atravesar las cabezas del primer músculo interóseo dorsal, donde forma el arco palmar profundo en su unión con la arteria cubital. En ese trayecto dorsal emite las ramas que nutren todo el dorso de la mano. Da vascularización al primer dedo, con la arteria principal del pulgar, y, después, emite una rama hacia el dorso del segundo dedo, la primera arteria metacarpiana dorsal. Por último, forma el arco dorsal del carpo, que discurre hasta anastomosarse con la arteria interósea posterior y con la rama dorsal cubital. De este arco surge un entramado arterial del cual surgen hacia distal las arterias metacarpianas dorsales $2^{\mathrm{a}}, 3^{\mathrm{a}}, 4^{\mathrm{a}}$ y $5^{\mathrm{a}}$.

Quaba y Davison exponen, en su artículo, el colgajo de dorso de la mano a flujo reverso, ${ }^{4}$ describiendo la variabilidad y complejidad de la vascularización de dicha zona anatómica. Las arterias metacarpianas $1^{\mathrm{a}}, 2^{\mathrm{a}}$ y $3^{\mathrm{a}} \mathrm{se}$ encuentran presentes aproximadamente un $95 \%$ de las veces. Sin embargo, las arterias metacarpianas dorsales $4^{\mathrm{a}}$ y $5^{\mathrm{a}}$ son más inconstantes, originándose del sistema palmar cuando no están presentes a nivel dorsal. ${ }^{5}$

El sistema vascular metacarpiano dorsal discurre hacia distal, hasta anastomosarse con el palmar, que proviene del arco palmar profundo, formando la arteria digital común. En esta unión, que se produce en el espacio entre las cabezas de los metacarpianos, es donde nace una arteria perforante comisural que se ramifica para irrigar la piel del dorso de la mano. ${ }^{6}$ Esa arteria perforante es la rama comunicante dorsal de la arteria digital común, conocida como perforante de Quaba. - Fig. 1. El flujo a través de dicha perforante puede ser anterógrado o retrógrado según provenga del arco dorsal o palmar, respectivamente.

Siguiendo la vascularización retrógrada del arco palmar es posible obtener un colgajo basado en la perforante de Quaba, aunque también es posible obtener un colgajo basado en una

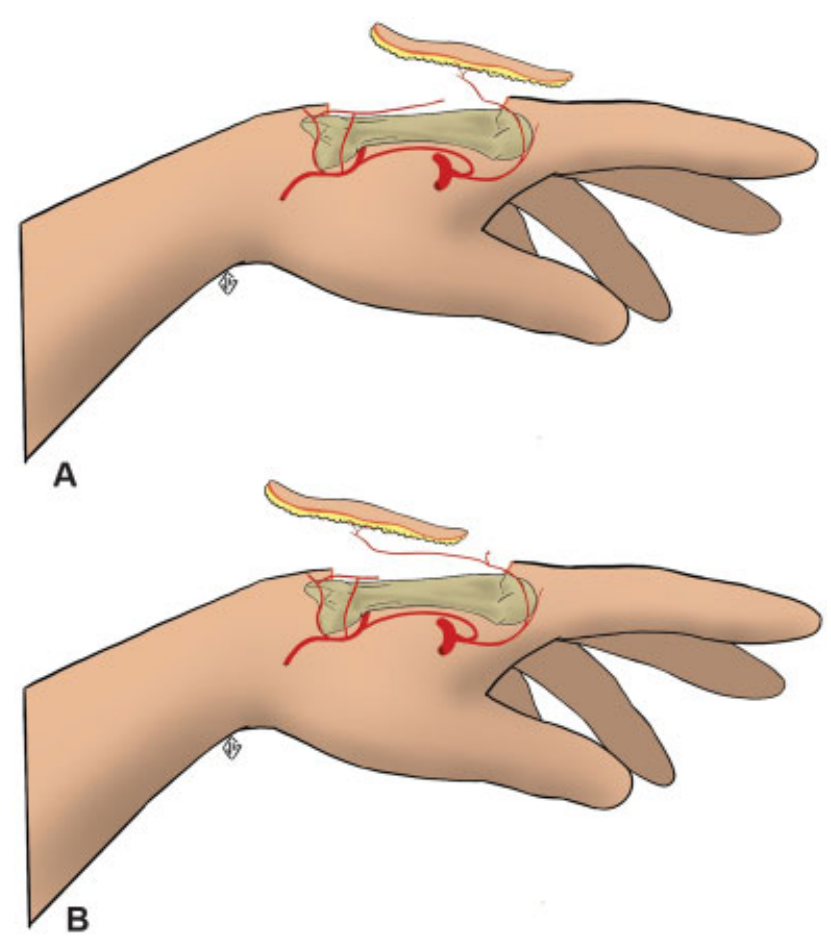

Fig. 1 Colgajo fasciocutáneo basado en perforante de arteria intermetacarpiana (IMCAP) a flujo reverso, basado en arteria perforante de Quaba (A) y perforante proximal con pedículo más largo (B).

perforante más proximal, obteniendo un pedículo más largo que puede alcanzar defectos aún más distales.

\section{Casos Clínicos}

\section{Caso Clínico 1}

Varón de 38 años que presenta un defecto de cobertura dorsal de PIP y F2 de $5^{\circ}$ dedo de mano izquierda, tras celulitis necrotizante de partes blandas y amplio desbridamiento de la zona afectada. El defecto expone el aparato extensor del $5^{\circ}$ dedo en zonas extensoras II y III, funcionalmente viables. Se decide realizar cobertura con un colgajo fasciocutáneo a flujo reverso, de la arteria intermetacarpiana dorsal (IMCAP), basado en la arteria perforante del $4^{\circ}$ espacio intermetacarpiano.

En la planificación del colgajo se sigue un patrón similar en los tres casos. En primer lugar, se buscan las arterias perforantes, mediante Doppler Probe, a lo largo del espacio intermetacarpiano elegido. Se selecciona la arteria perforante más distal y que presente mejor flujo. Y, por último, se realiza el diseño del colgajo teniendo en cuenta el arco de rotación de $180^{\circ}$ para lograr la cobertura del defecto.

En ese caso, el defecto de cobertura del tendón extensor no alcanza la $\mathrm{F} 3$ aunque sí existe una pérdida de sustancia susceptible de granulación, que no precisó extensión del colgajo basado en la arteria perforante comisural de falange proximal, ${ }^{7}$ y se solventó con un injerto de piel de antebrazo, evitando, así, la posible congestión venosa posquirúrgica. - Fig. 2 


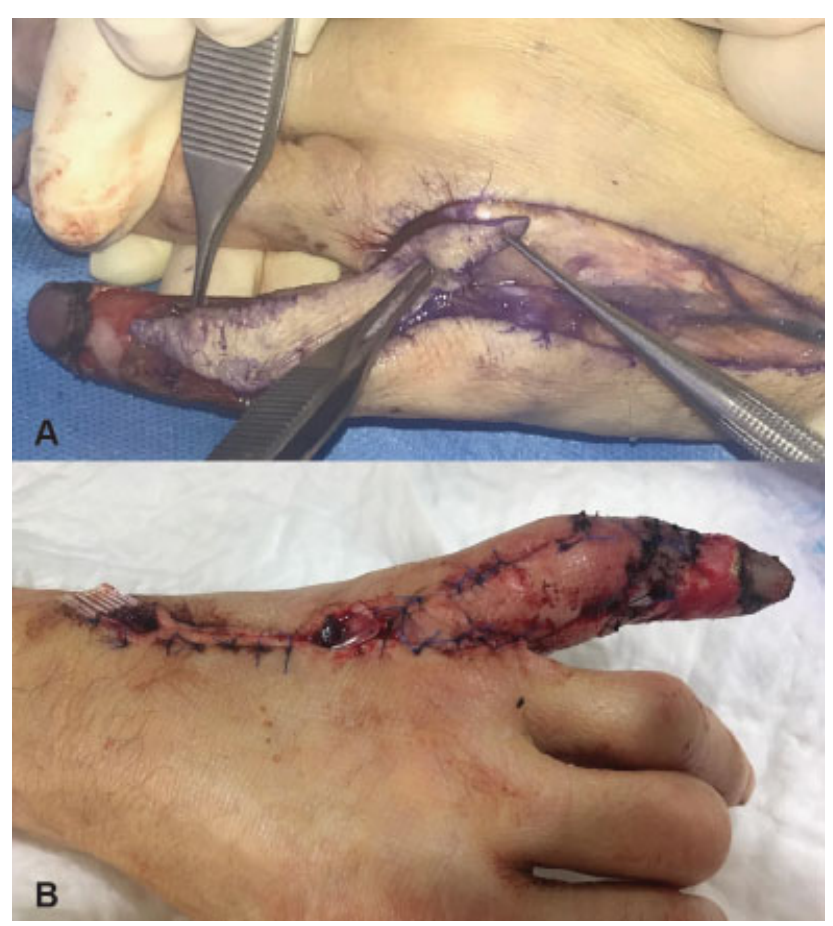

Fig. 2 (A) Colgajo IMCAP de $4^{\circ}$ espacio para cobertura. Detalle de rotación de $180^{\circ}$. (B) Postoperatorio inmediato.

De forma intraoperatoria, se realiza una prueba con Doppler vascular, tras la rotación y transposición del colgajo y de la arteria comisural perforante de la arteria intermetacarpiana dorsal, verificando el flujo correcto a través de ella.

La zona donante permite un cierre directo de la misma.

Se realiza inmovilización con una férula en extensión volar que limite la posibilidad de tracción del pedículo, la cual se mantiene durante 2 semanas.

El colgajo sufre congestión venosa distal, evidenciada a las 48 horas de la intervención, manejándose de forma conservadora con retirada parcial de sutura y elevación del miembro. El injerto cutáneo queda prendido en un periodo de 5-7 días y la zona donante no muestra complicaciones posoperatorias.

A las 4 semanas el defecto muestra una cobertura estable, con extensión MCF completamente conservada a 0 grados y limitación de la flexión a $60^{\circ}$. La extensión IFP no alcanza los $0^{\circ}$ y la flexión se mantiene estable a los $80^{\circ}$.

\section{Caso Clínico 2}

Varón de 76 años que sufre traumatismo, con maquinaria radial, en dorso de $3^{\circ}$ y $4^{\circ}$ dedos de mano izquierda. No presenta fracturas ni afectación del aparato extensor en ambos dedos. Se opta por un manejo conservador de la lesión en $3^{\circ}$ dedo, ya que no expone tejidos nobles.

En el dorso de PIP de $4^{\circ}$ dedo, y tras desbridamiento de tejidos no viables, se decide realizar cobertura del defecto creado de $3 \times 1 \mathrm{~cm}$, durante la misma intervención. En este caso, el colgajo se diseña en el $3^{\circ}$ espacio intermetacarpiano dorsal, asegurando así una cobertura de mayor fiabilidad vascular.

Se realiza inmovilización con una férula en extensión volar que limita la posibilidad de tracción del pedículo, y se mantiene durante las 2 primeras semanas de posoperatorio.

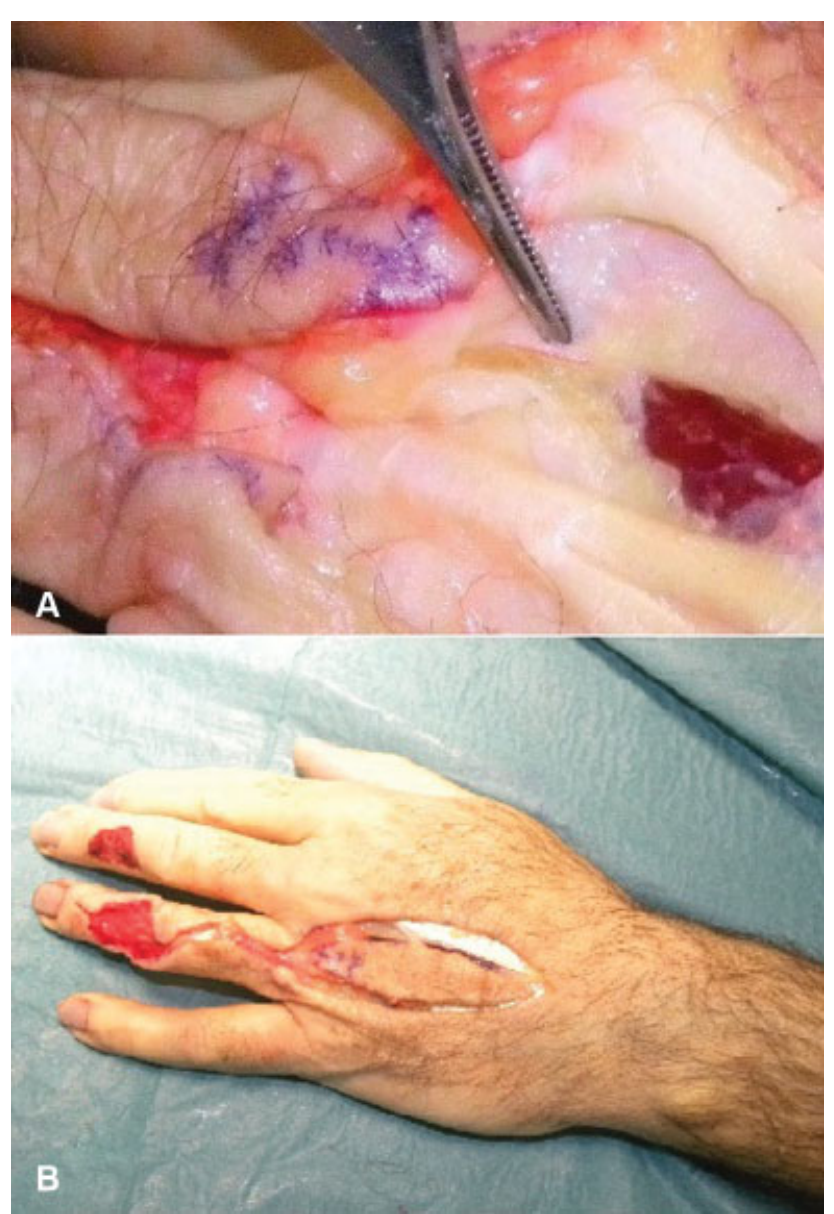

Fig. 3 (A) Detalle de la arteria perforante IMCAP. (B) Tallado de colgajo fasciocutáneo IMCAP de $3^{\circ}$ espacio intermetacarpiano.

El colgajo muestra edema perilesional a partir del primer día postoperatorio, sin signos de congestión venosa distal ni otras complicaciones.

La zona donante cierra de forma directa sin incidencias posoperatorias.

La flexión y la extensión de PIP alcanzan rangos normales tras 4 semanas de rehabilitación. - Fig. 3

\section{Caso 3}

Mujer de 64 años que sufre quemadura subdérmica, en dorso de $2^{\circ}$ dedo de mano izquierda, con aceite hirviendo, de $2 \times 2 \mathrm{cms}$. Tras curas seriadas con sulfadiazina argéntica realizamos limpieza y desbridamiento quirúrgico de escara necrótica, observando importante edema perilesional y exposición de aparato extensor en zona extensora III (PIP) con pérdida de peritenon correspondiente. Se decide realizar cobertura con colgajo IMCAP de $2^{\circ}$ espacio intermetacarpiano dorsal.

Se realiza inmovilización durante 2 semanas con una férula en extensión limitándose la posibilidad de tracción del pedículo.

A las 6 semanas de rehabilitación se ha recuperado la extensión de MCF a $5^{\circ}$ y flexión a $90^{\circ}$, muy similar a la movilidad basal previa de la paciente.

La zona donante se cierra de forma directa sin incidencias en el postoperatorio. - Fig. 4 


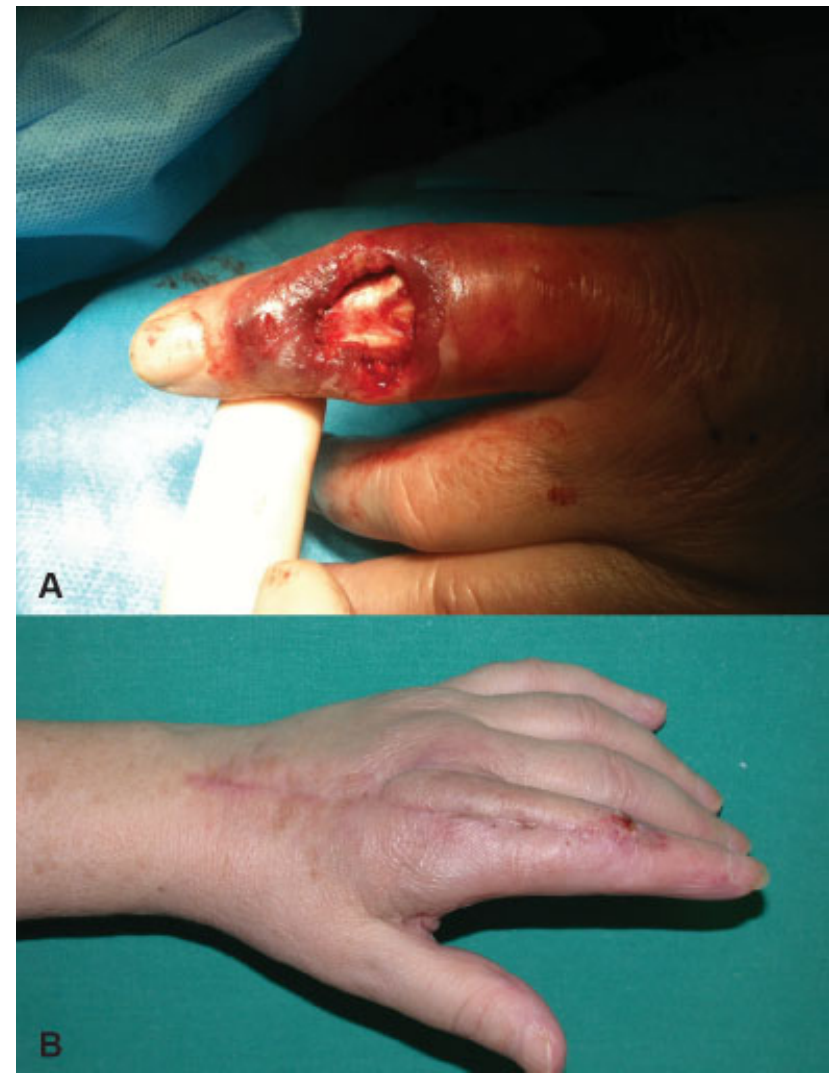

Fig. 4 (A) Defecto de cobertura dorsal de PIP de $2^{\circ}$ dedo con exposición tendinosa extensora. (B) Colgajo fasciocutáneo IMCAP de $2^{\circ}$ espacio intermetacarpiano dorsal.

\section{Discusión}

La cobertura de defectos en el dorso de la mano comprende numerosas opciones reconstructivas, desde injertos a colgajos regionales, e incluso colgajos libres, transferidos y anastomosados con técnicas de microcirugía.

La cobertura con injertos de piel sólo sería posible en caso de presentar un peritenon en perfecto estado, situación que en muchos casos es poco frecuente tras la lesión ocurrida. En nuestros casos, y tras un desbridamiento quirúrgico adecuado, la exposición de estructuras no permitió ese tipo de cobertura.

La cobertura mediante transferencia de colgajos libres puede tener sus indicaciones, pero parece excesivamente compleja en esa zona anatómica, especialmente en caso de poder recurrir a colgajos regionales con buenas condiciones de cobertura.

Las series más largas de la literatura muestran datos similares a las de este artículo, en cuanto a mecanismo de lesión, siendo las principales las traumáticas, quedando las infecciones y las quemaduras en segundo plano. ${ }^{8,9}$

Las principales características que hacen tan atractivo este colgajo con respecto a otras posibilidades de cobertura son las siguientes:

- Piel bien vascularizada. Es necesario un tejido vascularizado que asegure la cobertura del aparato extensor, óseo y articular subyacente, con una piel de similares características. Existen colgajos regionales musculares, que pueden ser útiles en determinadas ocasiones, pero los colgajos fasciocutáneos regionales son electivos por similitud tisular.

- Fiabilidad. Existen colgajos locales con muy buena fiabilidad vascular en lo que respecta a la vascularización del tejido transpuesto, doppleados previamente, y que no van a precisar, en la gran mayoría de las ocasiones, de revisiones quirúrgicas. Las tasas de complicación, habitualmente congestión venosa, son de $0 \%$ al $17 \%$ según series. ${ }^{8-12}$ En esas series se mezclan datos de colgajos con diseño extendido, con colgajos de diseño clásico, lo cual puede inducir a un sesgo al analizar los datos de posibles complicaciones.

La serie de Gregory y col., con 69 colgajos y un riesgo relativo de complicación $14 \%$ presenta 7 necrosis parciales y 3 pérdidas totales de colgajo. ${ }^{8}$ Así mismo, la serie de Sebastian y col., con 60 colgajos y un riesgo relativo de complicación del 17\%, muestra también 7 pacientes con congestión venosa y 2 pérdidas totales del colgajo y 1 infección relacionada con insuficiencia arterial (que se resolvió sin incidencias). ${ }^{9}$ La serie presentada aquí muestra datos diferentes que deberían analizarse con cautela dada la pequeña muestra de la serie. Además, conforme seguimos las series publicadas que van progresivamente aumentando de tamaño de muestra, van mostrando también un progresivo aumento de las complicaciones con tendencia a alcanzar las cifras de las series de Gregory y Sebastian. Es de esperar que con aumentos de tamaño muestral las series puedan compararse y mostrar mayor homogeneidad, tanto en lo que respecta a las pérdidas totales de colgajo, como a las parciales.

La principal solución que muestran los autores de las grandes series para las pérdidas parciales es la cobertura con un injerto de piel parcial. ${ }^{8,9,11}$ En la serie de Shen, se aplica un protocolo de seguimiento con inyecciones de heparina $(0,1 \mathrm{~mL}$ de Heparina en inyecciones repetidas sin especificar), en caso de hallar congestión venosa distal. ${ }^{13}$ Las congestiones distales de esa serie se resolvieron completamente con este protocolo. La serie de Katerinski y col., emplea con éxito la aplicación de sanguijuelas en el único caso de congestión venosa que muestra de los 7 que presenta en su serie. ${ }^{12}$ Otros autores prefieren optar por la retirada de algunos puntos de sutura y elevación del miembro, como relatan Balan y col. ${ }^{14}$

En la serie presentada en este artículo, se opta por un tratamiento conservador con curas convencionales sin inyección de heparina y con elevación de la mano y retirada de puntos, dado que las zonas de congestión venosa corresponden a menos del $90 \%$ de la superficie del colgajo, con buen resultado en ambos casos.

- Técnica sencilla. La realización del colgajo no precisa de una curva de aprendizaje prolongada, aunque la habilidad del cirujano y el conocimiento anatómico de la región son siempre condición sinequanon para el éxito de la técnica. De todas formas, aunque se recomienda iniciación en microcirugía, la curva de aprendizaje es sencilla.

- Tiempo quirúrgico reducido. El tallado e insetting del colgajo no requiere más de una hora quirúrgica. Las 
técnicas microquirúrgicas conllevan tiempos quirúrgicos más prolongados.

- Estancia hospitalaria mínima. Habitualmente se realiza en régimen de Cirugía Mayor Ambulatoria. Algunos autores proponen una hospitalización más prolongada en caso de realizar colgajos extendidos, por el mayor riesgo de congestión venosa, y de esa forma poder aplicar medidas en caso de presentarse alguna complicación. ${ }^{11,13}$

- Tiempo de recuperación reducido. Similar o menor que para un injerto cutáneo, y menor que si se emplean técnicas más complejas. Los datos al respecto del retorno a la actividad laboral, sin embargo, son difíciles de asociar al resultado de la técnica, dado que en muchos casos se asocia al periodo de baja que el paciente solicita por motivos ajenos a la evolución de la cobertura de su lesión. ${ }^{9}$

- Mínima morbilidad. No existen muchas publicaciones centradas en la morbilidad de esa técnica. Koch y col. publicaron una comparativa, con respecto al mismo dedo contralateral, con un estudio retrospectivo, del que ellos mismos aseguran que tiene muchos sesgos, pero que confirman como morbilidad baja. ${ }^{8}$ Además, comparan los datos de su estudio con el de otro con colgajos de dedos cruzados, otra alternativa sencilla para defectos similares. Koch encuentra que el colgajo de dedos cruzados presenta mayor morbilidad de zona donante y peor resultado estético. ${ }^{15}$

Respecto a la morbilidad sensitiva, con la correcta disección de las ramas dorsales del nervio cubital o radial, no se producirá ninguna pérdida funcional. Algunos autores, como Shen y col., proponen la inclusión de las ramas nerviosas dorsales (radiales o cubitales) en una variante técnica basada en la teoría de colgajos neurocutáneos propuesta por Bertelli y Khouri. ${ }^{16}$ La intención es preservar la vascularización asociada a las ramas nerviosas, enriqueciendo el aporte vascular, como explican en su artículo. ${ }^{13}$ De sus 14 pacientes, 2 presentaron hipoestesia en el seguimiento a largo plazo. Sin embargo, la serie de Shen no presenta mejores datos de complicación, con 5 pacientes con congestiones distales y 1 pérdida del colgajo. A la luz de los resultados obtenidos hasta el momento, se ha considerado preferible la identificación y preservación de las ramas sensitivas dorsales, que no aportarán beneficio a la supervivencia del colgajo. De todas formas, es posible que en ocasiones la rama atraviese el colgajo y sea irremediable la sección de la misma. ${ }^{9}$

Por último, respecto a la zona donante, lo ideal es que pueda cerrarse de forma directa y sin tensión, y eso puede preverse desde el diseño del colgajo. La serie más larga hasta la fecha no menciona nada al respecto, ${ }^{8}$ sin embargo, la de Sebastian, la segunda más numerosa, indica que precisa de injerto de piel parcial para la cobertura de la zona donante de hasta 5 pacientes (9\%), especificando que colgajos de más de $3 \mathrm{~cm}$ de ancho precisarán cobertura con injerto.

\section{Conclusión}

Los colgajos regionales para cobertura del dorso de los dedos son de elección en la reconstrucción de dicha zona anatómica.
El colgajo tipo Propeller, de perforantes IMCAP de dorso de la mano, aporta piel vascularizada de similares características, con suficiente fiabilidad y mínima morbilidad. Es una técnica sencilla y conlleva un tiempo quirúrgico reducido, una estancia hospitalaria mínima y un tiempo de recuperación asumible. Todo ello debe tenerse en cuenta en la evaluación de los costesefectividad y de la productividad laboral. La serie presentada aquí muestra características que continúan la tendencia de las principales series al respecto de ese tipo de reconstrucción cuya principal complicación en cuanto a frecuencia es la congestión venosa.

Conflictos de interés

Niguno

\section{Bibliografía}

1 Fernandez-García A, Soria-Cogollos T. Revisión clínica de diez técnicas tradicionales para cobertura de lesiones en dedo. Cir Plast Iberolatinoam 2007;33:3

2 Merle M, Dautel G Cirugía de la mano, Urgencias. Ciudad Autónoma de Buenos Aires. 2017

3 Zaidenberg C, Angrigiani C. Colgajos reversos para el tratamiento de las heridas graves de los dedos. Rev Asoc Argent Ortop Traumatol 1995;58(01):58-65

4 Quaba AA, Davison PM. The distally-based dorsal hand flap. Br J Plast Surg 1990;43(01):28-39

5 Omokawa S, Tanaka Y, Ryu J, Kish VL. The anatomical basis for reverse first to fifth dorsal metacarpal arterial flaps. J Hand Surg [Br] 2005;30(01):40-44

6 Bailey SH, Andry D, Saint-Cyr M. The dorsal metacarpal artery perforator flap: a case report utilizing a quaba flap harvested from a previously skin-grafted area for dorsal 5 th digit coverage. Hand (N Y) 2010;5(03):322-325

7 Pelissier P, Casoli V, Bakhach J, Martin D, Baudet J. Reverse dorsal digital and metacarpal flaps: a review of 27 cases. Plast Reconstr Surg 1999;103(01):159-165

8 Gregory H, Heitmann C, Germann G. The evolution and refinements of the distally based dorsal metacarpal artery (DMCA) flaps. J Plast Reconstr Aesthet Surg 2007;60(07):731-739

9 Sebastin SJ, Mendoza RT, Chong AK, et al. Application of the dorsal metacarpal artery perforator flap for resurfacing soft-tissue defects proximal to the fingertip. Plast Reconstr Surg 2011;128 (03):166e-178e

10 Wang P, Zhou Z, Dong Q Jiang B, Zhao X. Reverse second and third dorsal metacarpal artery flap for repair of distal and middle segment finger soft tissue match. J Reconstr Microsurg 2011;27:495-502

11 Koch H, Bruckmann L, Hubmer M, Scharnagl E Extended reverse dorsal metacarpal artery flap: clinical experience and donor site morbidity Journal of Plastic, Reconstructive \& Aesthetic Surgery (2007) 60.

12 Katerinaki E, Chakrabarty KH. Distally based dorsal metacarpal flaps: a review of a series of patients treated in a 6 -month period. Injury 2004;35(11):1176-1181

13 Shen H, Shen Z, Wang Y, Zhang K, Zhang Z, Dai X. Extended reverse dorsal metacarpal artery flap for coverage of finger defects distal to the proximal interphalangeal joint. Ann Plast Surg 2014;72 (05):529-536

14 Balan JR, Mathew S, Kumar P, et al. The reverse dorsal metacarpal artery flap in finger reconstruction: A reliable choice. Indian J Plast Surg 2018;51(01):54-59

15 Koch H, Kielnhofer A, Hubmer M, Scharnagl E. Donor site morbidity in cross-finger flaps. Br J Plast Surg 2005;58(08):1131-1135

16 Bertelli JA, Khoury Z. Neurocutaneous island flaps in the hand: anatomical basis and preliminary results. Br J Plast Surg 1992;45 (08):586-590 